\title{
EVALUATION AND QUALITY CONTROL OF NASAL SPRAY
}

\author{
Patil Vidya Bankar ${ }^{1}$, Kalkotwar RS ${ }^{* 1}$, Patel Ankita ${ }^{1}$, Tathe Swati ${ }^{1}$, Jadhav VB ${ }^{2}$ \\ ${ }^{1}$ Dept. of Quality Assurance, S.N.D. College of Pharmacy, Yeola, Nashik, 423401, India \\ ${ }^{2}$ Dept. of Pharma.Analy sis, S.N.D., College of Pharmacy, Yeola, Nashik, 423401, India \\ *Corresponding author-E-mail Id: rameshkalkotwar@gmail.com
}

Received 23 May 2012; Review Completed 10 July 2012; Accepted 10 July 2012, Available online 15 July 2012

\begin{abstract}
Nasal drug delivery has now been recognized as a very promising route for delivery of therapeutic compounds including biopharmaceuticals. This route is also advisable for drugs undergoing extensive first pass effect. The present article highlights the evaluation parameters of nasal spray, suspension, and solutions. While formulat ing the Nasal drug delivery formulations various parameters are to be consider such as Appearance, Color, and Clarity, Identification, Drug content (Assay) but these are not just sufficient for efficacy and bioavailability of drug. Very little is known to the researchers about other parameters of quality control of Nasal Spray. The same are elaborated in this article with acceptance crateria.
\end{abstract}

Key words: Nasal spray, Evaluation, Quality control, Drug delivery system.

\section{INTRODUCTION}

Currently, Nasal drug delivery has been recognized as a very promising route for delivery of therapeutic compounds including biopharmaceuticals. Nasal administration is a logical choice for topical nasal treatments such as antihistamines and corticosteroids. The nasal mucosa has also received attention as a viable means of systemic administration of analgesics, sedatives, hormones, cardiovascular drugs, and vaccines. Conventionally, the nasal route has been used for local delivery of drugs for treating nasal allergy, nasal congestion, or nasal infections. However systemic delivery through the nasal route has recently begun to explore possibilities for those requiring a rapid onset of action or necessitating avoidance of severe proteolysis involved in oral administration (e.g., most peptide and protein drugs). Successful attempts to deliver corticosteroid hormones through the nasal route for systemic absorption have triggered further studies in this area. ${ }^{1-3}$

Researchers have studied the anatomical and physiological aspects of the nasal membrane, including its vascular nature, as they relate to drug delivery. ${ }^{4-11}$ There are three distinct functional regions in the nose- the vestibular, respiratory, and olfactory. Among these, the respiratory region is the most important for systemic drug delivery. ${ }^{12}$ The respiratory epithelium consists of basal, mucuscontaining goblet, ciliated columnar and non-ciliated columnar cell types. ${ }^{13}$ The cilia move in a wavelike fashion to transport particles to the pharynx area for ingestion. ${ }^{14}$ Additionally, the cells in this region are covered by nearly 300 microvilli, providing a large surface area for absorption. Below the epithelium is the lamina propria. This is where blood vessels, nerves, serous glands, and mucus secretary glands may be found. The lamina propria also houses a dense network of capillaries, through which drug absorption takes place. The nasal passage epithelium is covered by a mucus layer that is renewed every 10 to 15 minutes. ${ }^{15}$ The $\mathrm{pH}$ of the mucosal secretions ranges from 5.5 to 6.5 in adults and 5.0 and 6.7 in children. ${ }^{16}$ The (c) 2011, JDDT. All Rights Reserved mucus layer entraps particles, which are then cleared from the nasal cavity by the cilia. ${ }^{17}$

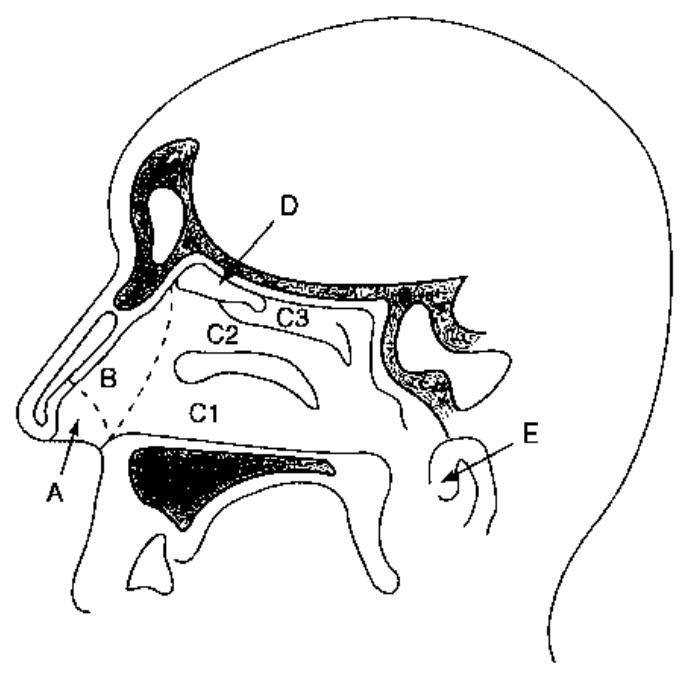

Figure 1: Schematic of a sagittal section of human nasal cavity showing the nasal vestibule (A), atrium (B), respiratory region: inferior turbinate $(\mathrm{C} 1)$, middle turbinate $(\mathrm{C} 2)$ and the superior turbinate (C3), the olfactory region (D) and nasophary nx (E) ${ }^{18}$

\section{Nasal Sprays}

The following test parameters are recommended for nasal spray drug products. Appropriate acceptance criteria and validated test procedures should be established for each test parameter.

\section{EVALUATION PARAMETERS :}

(For Nasal Spray dosage form - Inhalation Solutions, Suspensions, and $S$ prays $)^{19-22}$

\section{a. Appearance, Color, and Clarity:}

The appearance of the content of the container (i.e., formulation) and the container closure system (e.g., pump components, inside of the container) should conform to 
their respective descriptions as an indication of the drug product integrity. If any color is associated with the formulation (either present initially or from degradative processes occurring during shelf life) then a quantitative test with appropriate acceptance criteria should be established for the drug product by the manufacturer.

\section{b. Identification:}

A specific identification test(s) is recommended to verify the identity of the drug substance in the drug product. Chromatographic retention time alone is not an adequate method to ensure the identity of the drug substance in the drug product. If the drug substance is a single enantiomer, then at least one of the methods should be specific for this property.

\section{c. Drug Content (Assay):}

The assay of drug substance in the entire container should be determined analytically with a stability indicating procedure. This test provides assurance of consistent manufacturing (e.g., formulation, filling, sealing). The acceptance criteria ( assay limits as specified in official books) should be tight enough to ensure conformance in other related attributes (e.g., spray content uniformity). A suitable assay procedure should be designed to address any degradation of the drug substance, adherence of the drug substance to the container and closure components, and the potential effect of formulation evaporation and/or leakage.

\section{d. Impurities and Degradation Products :}

The levels of degradation products and impurities should be determined by means of stability indicating procedure(s). Acceptance criteria should be set for individual and total degradation products and impurities. For identification and qualification thresholds, refer to the appropriate guidance. All related impurities appearing at levels of 0.1 percent or greater should be specified. Specified impurities and degradation products are those, either identified or unidentified, that are individually listed and limited in the drug product specification.

\section{e. Preservative(s) and Stabilizing Excipient(s) Assay:}

If preservatives, antioxidants, chelating agents, or other stabilizing excipients (e.g., benzalkonium chloride, phenylethyl alcohol, edetate) are used in the formulation, there should be a specific assay for these components with associated acceptance criteria (At a concentration of 0.10 percent or 1.0 milligram per day).

\section{f. Pump Deli very:}

A test to assess pump-to-pump reproducibility in terms of drug product performance and to evaluate the metering ability of the pump should be performed. The proper performance of the pump should be ensured primarily by the pump manufacturer, who should assemble the pump with parts of precise dimensions. Pump spray weight delivery should be verified by the applicant for the drug product. In general, pump spray weight delivery acceptance criteria should control the weight of the individual sprays to within \pm 15 percent of the target weight and their mean weight to within \pm 10 percent of the target weight.

\section{g. S pray Content Unifor mity (SCU):}

The spray discharged from the nosepiece should be thoroughly analyzed for the drug substance content of multiple sprays from an individual container, among containers, and among batches of drug product. This test should provide an overall performance evaluation of a batch, assessing the formulation, the manufacturing process, and the pump. The number of sprays per determination should not exceed the number of sprays per single dose. A single dose represents the minimu nu mber of sprays per nostril specified in the product labeling. To ensure reproducible in vitro dose collection, the procedure should have controls for actuation parameters (e.g., stroke length, depression force). The test may be performed with units primed following the instructions in the labeling. The amount of drug substance delivered from the nosepiece should be expressed both as the actual amount and as a percent of label claim. This test is designed to demonstrate the uniformity of medication per spray (or minimum dose), consistent with the label claim, discharged from the nosepiece, of an appropriate number ( $\mathrm{n}=10$ is recommended) of containers from a batch. The primary purpose is to ensure SCU within the same container and among multiple containers of a batch. The following acceptance criteria are recommended:

$>$ The amount of active ingredient per determination is not outside of 80-120 percent of label claim for more than 1 of 10 containers, none of the determinations is outside of 75-125 percent of the label claim, and the mean is not outside of 85-115 percent of label claim.

$>$ If 2 or 3 of the 10 determinations are outside of 80 120 percent of the label claim, none is outside of 75125 percent of label claim, and the mean is not outside of 85-115 percent of label claim, an additional 20 container should be sampled (second tier). For the second tier of testing of a batch, the amount of active ingredient per determination is not outside of 80-120 percent of the label claim for more than 3 of all 30 determinations, none of the 30 determinations is outside of 75-125 percent of label claim, and the mean is within $85-115$ percent of label claim.

\section{h. S pray Content Unifor mity (SCU) through container life:}

The purpose of this test is to assess whether the product delivers the labeled number of full medication sprays meeting SCU acceptance criteria throughout the life of the nasal spray unit. The test involves determining the SCU from the beginning of unit life and at the label claim number of sprays per container for an appropriate nu mber of containers ( $\mathrm{n}=5$ is recommended). The following acceptance criteria are recommended.

$>$ The amount of active ingredient per determination is not outside of 80-120 percent of label claim for more than 1 of 10 determinations from five containers, none of the determinations is outside of 75-125 percent of the label claim, and the means for each of the beginning and end determinations are not outside of 85-115 percent of label claim.

$>$ If 2 or 3 of the 10 determinations are outside of 80 120 percent of the label claim, none is outside of 75125 percent of label claim, and the means for each of 
the beginning and end determinations are not outside of 85-115 percent of label claim, an additional 10 containers are sampled at the beginning of unit life and at the label claim number of sprays (second tier). For the second tier of testing of a batch, the amount of active ingredient per determination is not outside of $80-120$ percent of the label claim for more than 3 of all 30 determinations, none of the 30 determinations is outside of 75-125 percent of label claim, and the means for each of the beginning and end determinations are not outside of $85-115$ percent of label claim

\section{i. Spray Pattern and Plume Geometry:}

Characterizations of spray pattern and plume geometry are important for evaluating the performances of the pump and nozzle. Various factors can affect the spray pattern and plume geometry, including the size and shape of the nozzle, the design of the pump, the size of the metering chamber, and the characteristics of the formulation. Spray pattern testing should be performed on a routine basis as a quality control for release of the drug product. However, the characterization of plume geometry should typically be established during the characterization of the product and is not necessarily tested routinely thereafter. The proposed test procedure for spray pattern, including analytical sampling plans, should be provided in detail to allow duplication by agency laboratories. For example, in the evaluation of the spray pattern, the spray distance between the nosepiece and the collection surface, number of sprays per spray pattern, position and orientation of the collection surface relative to the nosepiece, and visualization procedure should be specified. The acceptance criteria for spray pattern should include the shape (e.g., ellipsoid of uniform density) as well as the size of the pattern (e.g., no axis is greater than $x$ millimeters and the ratio of the longest to the shortest axes should lie in a specified range, for example, 1.00-1.20). The spray pattern should be determined, preferably by a procedure specific for the drug substance, at different distances (e.g., two) from the nosepiece to provide greater discriminatory capability to the test. Variability in the test can be reduced by the development of a sensitive detection procedure and by providing procedure 502 specific training to the analyst.

\section{j. Droplet Size Distribution:}

For both suspension and solution nasal sprays, the specifications should include an appropriate control for the droplet size distribution (e.g., 3 to 4 cut-off values) of the delivered plume subsequent to spraying under specified experimental and instrumental conditions. Appropriate and validated dynamic plume droplet size analytical procedures should be described in sufficient detail to allow accurate assessment by Agency laboratories (e.g., apparatus and accessories, software version and calculation algorithms, sample placement, laser trigger condition, measurement range, beam width).

\section{k. Par ticle Size Distributi on (Suspensions):}

For suspension nasal sprays, the specification should include controls for the particle size distribution of the drug substance particles in the formulation. This quantitative procedure should be appropriately validated in terms of its sensitivity and ability to detect shifts that may occur in the distribution. The acceptance criteria should control the complete distribution and should reflect the data obtained for the submitted batches (e.g., clinical, preclinical, biobatch, primary stability, production).

\section{Microscopic Evaluation (Sus pensions):}

This test, which involves a qualitative and semi quantitative microscopic examination of the suspension formulations, is complementary to partic le size distribution testing for both release and stability purposes. For example, the examination provides information on the presence of large particles and changes in morphology of the drug substance particles, extent of agglomerates, and crystal growth. Additionally, where changes in the solid state of the drug substance can affect the bioavailability, performance, stability, or other properties of the drug product, microscopic evaluation or other appropriate procedures are recommended to control and monitor changes that are observed on stability.

\section{m. Foreign Particulates :}

For both solution and suspension nasal sprays, there should be validated tests and associated acceptance criteria for foreign particulates. Foreign particulates may orig inate during manufacturing, from formu lation components, and, in particular, from the container and closure components. Levels of foreign particulates in the drug product may increase with time, temperature, and stress.

\section{n. Microbial Limits :}

The microbial quality should be controlled by appropriate tests and acceptance criteria for total aerobic count, total yeast and mold count, and freedom from designated indicator pathogens. Acceptance criteria should be reflective of the data for the submitted batches (e.g., clinical, preclinical, biobatch, primary stability, production), but at a minimum should meet the recommended microbial limits acceptance criteria in USP $<1111>$, Microbiological Attributes for Non-sterile Pharmacopeial Articles. Furthermore, appropriate testing should show that the drug product does not support the growth of microorganis ms and that microbiological quality is maintained throughout the expiration dating period. For a description of this test, refer to the procedure in USP $<61>$.

\section{o. Preservative Effectiveness:}

For nasal sprays that contain a preservative(s), stability testing should include microbial challenge studies performed on the first three production batches of drug product.

\section{p. Net Content and Weight Loss (Stability):}

Nasal spray drug products should include acceptance criteria for net content and weight loss on stability. Since storage orientation plays a key role in any weight loss, the drug product should be stored in upright and inverted or upright and horizontal positions to assess this characteristic.

The total net content of all formulation components in the entire container should be determined. The net content of 
each of 10 test containers should be in accordance with the release specification. For a description of this test, refer to the procedure in USP Chapter <755> Minimu m Fill.

\section{q. Leachables (Stability):}

The drug product should be evaluated for compounds that leach from elastomeric or plastic components of the container closure system, such as nitrosamines, monomers, plasticizers, accelerators, antioxidants, and vulcanizing agents. The development of appropriate analytical procedures to identify, monitor, and quantify the leached components in the drug product should be done during investigational studies. These validated procedures can, in turn, be used for testing of the drug product throughout the expiration dating period. Appropriate acceptance criteria for the levels of leached compounds in the formulation should be established.

\section{r. pH:}

For both solution and suspension nasal sprays, the apparent $\mathrm{pH}$ of the formulation should be tested and an appropriate acceptance criterion established. Lysozyme is found in nasal secretions, which is responsible for destroying certain bacteria at acidic $\mathrm{pH}$. Under alkaline conditions, lysozyme is inactivated and the nasal tissue is susceptible to microbial infection. It is therefore advisable to keep the formulation at a $\mathrm{pH}$ of 4.5 to 6.5 keeping in mind the physicochemical properties of the drug as drugs are absorbed in the un-ionized form ${ }^{23}$

\section{s. Osmolality:}

The osmolality of the formulation should be tested and controlled with an appropriate procedure and acceptance developed by manufacturer.

\section{CONCLUSION:}

The quality control of Nasal Spray is critical area where high standards are to be maintained therefore evaluation of different parameters discussed in this review shows strict pharmacovigilance as far as such type of dosage forms are concerned. Impurities and Degradation Products, Preservative(s) and Stabilizing Excipient(s) Assay, Pump Delivery, Spray content uniformity, Spray Content Uniformity (SCU) through Container Life, Spray Pattern and Plume Geometry, Droplet Size Distribution, Particle size distribution (suspension), Microscopic Evaluation (Suspensions), Foreign Particulates, Microbial limit, Preservative Effectiveness, Net Content and Weight Loss (Stability), Leachables (Stability), PH, Osmolality. The acceptance criteria for these parameters have been recognized by officials books all over the world. The attempts to deliver corticosteroid hormones through the nasal route for systemic absorption have triggered further studies and strict control over the delivered dosage.

\section{REFERENCES :}

1. Ugwoke. MI, Agu. RU, Verbeke N, Kinget R. Nasal mucoadhesive drug delivery: Background, applications, trends and future perspectives', Advanced Drug Del Reviews. 2005; 57: 1640-1665

2. Costantino HR., Lisbeth I, Brandt G. Johnson PH, Quay SC. Intranasal delivery: Physicochemical and therapeutic aspects. International Journal of Pharmaceutics. 2007; 337: 1-24

3. Illum Lisbeth. Nasal drug delivery possibilities, problems and solutions. J Control Release.2003; 87:87-198

4. My gind N. Scanning electron microscopy of the human nasal mucosa. Rhinology 1975; 13: 57-75.

5. Watanabe K, Watanabe I, Saito Y, Mizuhira V. Characteristics of capillary permeability in nasal mucosa. Ann Otol Rhinol Laryngol 1980; 89: 377-382.

6. Geurkink N. Nasal anatomy, physiology, and function. J Allergy Clin Immunol 1983; 72: 123-128.

7. Brofeldt S, Secher C, My gind N. Biophy sical characteristics of nasal secretions: A preliminary report. Eur J Respir Dis 1983; 64: 436-440.

8. Bende M. Studies of blood flow in the human nasal mucosa. Eur J Respir Dis 1983; 64: 400-402.

9. Bisgaard H, Krogs gaard O, Mygind N. Measurement of secretion in nasal lavage. Clin Sci 1987; 73: 217-222.

10. Dawes JDK, Prichard M. Studies of the vascular arrangement of the nose. J Anat 1953; 87: 311-322.

11. Cauna N, Hinderer KH. Fine structure of blood vessels of the human nasal respiratory mucosa. Ann Otol Rhinol Laryngol 1969; 78: 865-885.

12. Illum L. Transport of drugs from the nasal cavity to the central nervous system. Eur J Pharm Sci 2000; 23,11-18.

13. Schipper NG, Verhoef JC, Merkus FW. The nasal mucocilliary clearance: relevance to nasal drug delivery. Pharm Res 1991; 8: 807-814.

14. Mathison S, Nagilla R, Kompella UB. Nasal route for direct delivery of solutes to the central nervous system: fact or fiction. J Drug Target 1998; 5: 415-441.

15. Chien YW, Chang SF. Intranasal drug delivery for systemic medication. Crit Rev Ther Drug Carrier Syst 1987; 4: 67-194.
16. Hehar SS, Mason JD, Stephen AB, et al. Twenty four hour ambulatory nasal pH monitoring. Clin Otolaryngol 1999; 24: 24-25.

17. Mygind N, Anggard A. Anatomy and physiology of the nose pathophysiology alterations in allergic rhinitis. Clin Rev Allergy 1984; 2: 173-188.

18. M.I. Ugwoke, N. Verbeke, R. Kinget, The Biopharmaceutical Aspects of Nasal Mucoadhesive Drug Delivery, J. Pharm. Pharmacol. 2001,53, 3-21.

19. Guidance for Industry, Nasal spray \& Inhalation solution, Suspension \& Spray Drug Products, X:ICDERGUIDL2836DFT.WPD,M ay 1999 CMC.

20. USP/NF The Official Compendia Of Standards, Asian Edition 2005, 2304, 2681-2682.

21. Indian Pharmacopoeia 2010,vol II, ,Published by Government of India Ministry of Health and Family Welfare,New Delhi \& The Indian Pharmacopoeia commission, Ghaziabad, P.743.

22. British Pharmacopoeia 1998,vol II, Published on recommendation of medicines commission in pursuant to the Medicines Act 1968, P.1437-1439.

23. Thomsan R., Lysozyme and its relation to antibacterial properties of various tissues and Secretions. Arch Pathol 1940; 30:1096. 